\title{
Le voyage comme déplacement, la digression comme placement...
}

\section{Résumé:}

Dans cet article, notre analyse part du constat que le voyage -le déplacement- s'accompagne de digression -placement textuel et discursifschez Michel Tournier. Ce constat a engendré une réflexion sur les créations de l'imaginaire, une manière de concevoir le voyage et l'écriture du voyage, et comment le voyage provoque la digression. Somme toute, l'écriture du voyage et l'écriture de la digression se retrouvent enchevêtrés dans une complémentarité au profit tantôt de l'un tantôt de l'autre.

$$
\begin{aligned}
& \text { في هذا المقال، يبدأ تحليلنا من ملاحظة أن : }
\end{aligned}
$$

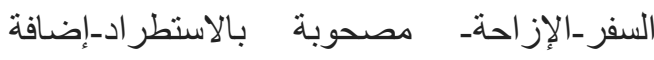

$$
\begin{aligned}
& \text { زوائد معرفية_عند الكاتب ميشال تورنيب. هذه بهابه } \\
& \text { الملاحظة أدت إلى تساؤلات و تفكير عن كيفية } \\
& \text { تخيل و ابتكار السفر، كتابة السفر أو الرحلة } \\
& \text { وبالأخص كيف للسفر أن يثير الاستطر اد. و كيف } \\
& \text { هي العلاقة لكتابة السفر و كتابة الاستطراد } \\
& \text { متشابكة ومتكاملة أحيان لصالح الواحدة و أحيان } \\
& \text { الأخرى. }
\end{aligned}
$$

\section{LABANI Ahlem}

Département de français Université des Frères Mentouri Constantine

\section{Introduction : \\ L'écriture d'un roman s'érige de l'imaginaire créatif de l'écrivain. Ce qui est plus qu'évident est que le roman ne fait pas que raconter une ou des histoires, mais au travers de cette ou ces histoires, il informe, explique et argumente. C'est le lieu de rencontre par excellence des divers genres littéraires et de plusieurs types de discours.}


Un genre protéiforme qui prend des aspects très variés, car il est structuré de façon complexe: son organisation, ses visées et les diverses séquences qu'il intègre.

Par ailleurs, dans un roman, l'auteur crée sa propre langue, choisit un certain style, une manière d'organiser l'histoire, de construire les phrases, d'utiliser et de choisir les mots. Il utilise et crée ses propres techniques d'écriture.

Justement, certains romans expliquent, informent, commentent lorsqu'ils abordent des domaines supposés peu connus du lectorat. Aussi lorsqu'une fiction, un univers sont construits, ils n'hésitent pas dans les explications de phénomènes, de comportements, de mécanismes, de géographie, d'histoire, de psychologie, de psychanalyse, d'anthropologie... Le texte devient donc un univers de savoir qui ouvre lieu à des digressions.

La digression est rupture avec la continuité narrative, c'est l'action de sortir du sujet principal dans un discours ou un récit et faire diversion, développer un sujet qui s'éloigne du sujet principal. Elle peut être de tout genre du discours seulement, toujours un genre différent de celui dont elle s'écarte.

Donc, dans l'acte même de digresser, il y a l'action de l'éloignement, de distanciation, d'aller vers quelque chose de nouveau, de différent. Bref, un changement.

De même, le voyage représente pour chacun un moyen d'évasion, d'échapper à quoi ? à qui ? Notamment échapper à la réalité vécue, à son destin, au présent, à ce qui est tangible, clair, palpable, tracé, évident, prévisible et partir à la recherche de quelque chose de nouveau, imprévisible, insaisissable, inconnu, à la recherche de l'oubli, de l'inconnu, du mystère ou alors c'est l'inverse. Parfois, on ne se reconnait pas dans le monde où l'on vit, on part à la recherche de soi-même, de lieu en lieu, d'expérience en expérience pour savoir qui l'on est, à quoi ressemble-t-on, de quoi sommes-nous fait?

On part en voyage dans des buts différents, on entreprend des parcours différents, on ressent des choses toutes aussi différentes les unes des autres mais un point est commun celui de la recherche, la découverte : de soi-même, des autres.

Chez Michel Tournier, le voyage est un vécu; qu'il soit réel ou imaginaire. Réel parce qu'il a voyagé beaucoup depuis son enfance-Michel Tournier a été imprégné de façon précoce par des lieux divers- ; imaginaire, à travers ses lectures (parce qu'il a voyagé avec les héros des textes lus) et a fait voyagé ses lecteurs à travers ses fictions.

Dans son premier texte romanesque, Vendredi ou les limbes $d u$ Pacifique, c'est la grande aventure pour Robinson qui part à la recherche de nouvelles terres depuis le Royaume Uni jusqu'en Amérique Centrale. Aventure du héros mais aussi celle du lecteur. Arrivé sur l'île, il se déplace encore; il 
explore toute l'île les mois suivants son naufrage. Et il va par la suite essayer de la cartographier. Pour cela, il se déplace graduellement tout en essayant de déchiffrer son écosystème, de comprendre son ordre naturellement établi. La faune et la flore constituent les matières premières à ses premières réflexions qui vont le conduire à des méditations profondes sur soi-même, sur ce qu'il a été, sur le monde environnant et sur l'Autre. Il y a déplacement corporel et imaginaire ce qui va donner lieu à des placements discursifs, des digressions. Tous les questionnements de Robinson, ses réflexions, ses méditations, ses remises en question ouvrent le champ à des digressions qui vont se placer dans le texte.

Un autre texte, celui de Les Météores, répond à ce même constat. Les héros, des frères jumeaux Jean et Paul, font le tour du monde, c'est le déplacement par excellence. Trahi par son frère jumeau, Jean part seul en voyage de noce. Paul n'acceptant pas cette séparation, cette gémellité dépariée, va à la recherche de son frère-pareil et débarque sur Venise. De là commence une course poursuite entre les deux frères qui vont faire un tour du monde. Ces déplacements à travers les pays, les continents, les cultures, les visions du monde, constituent un environnement plus que fertile pour des placements textuels, des digressions sur tout et tout le temps. De l'Italie à l'Allemagne, en passant par la Tunisie, l'Islande, le Japon, le Canada, les héros n'arrêtent pas de se déplacer même dans un même pays. Ils visitent, explorent, cherchent à comprendre, remettent en questions tout leur passé, leur savoir, leur vision des choses et des êtres. Les digressions ne manquent pas, elles les accompagnent dans leurs déplacements.

Dans La Goutte d'or, le héros Idriss, un jeune adolescent berbère algérien, se laisse photographier par une touriste parisienne qui part avec son image. Tout son entourage met Idriss en garde contre le pouvoir maléfique de l'image qui peut aller jusqu'à la mort. Afin de s'affranchir, il doit récupérer sa photo. C'est pourquoi il entreprend son périple pour la trouver, depuis son oasis natale Tabelbala dans la Sahara algérien jusqu'à Paris. Son itinéraire commence depuis Tabelbala puis Sidi Bel Abbès, Oran, Marseille et enfin Paris. A chaque nouvelle étape, Idriss est plongé dans un nouveau monde, lui qui n'a jamais quitté son oasis, et donc chaque déplacement constitue une nouvelle expérience enrichissante sur la vie, les hommes, les femmes, le monde les différentes conceptions et visions notamment sur l'image. Ainsi cela convoque des digressions multiples et variées.

Eléazar ou la Source et le Buisson est un texte où le déplacement alimente la narration. Le parcours d'Eléazar est son destin. Il se voit en la figure de Moïse et croit que leur destin est lié, son aventure personnelle s'éclaire à la lumière du destin de Moïse. Eléazar est un pasteur qui vivait dans son Irlande natale. Suite à la grande famine de 1845, il embarque avec sa femme et ses deux enfants pour le Nouveau Monde - la Terre Promise (le mot 
Californie qu'il entend pour la première fois, sonne à ses oreilles Canaan). D'ailleurs, la traversée en bateau jusqu'en Amérique dure quarante jours, qui rappellent les quarante jours et quarante nuits que Moïse a passé à la montagne. Les textes sacrés débordent; à chaque étape de son voyage, il ouvre sa Bible qui devient son guide ; chaque décision prise durant leur voyage et surtout dans le désert est une nouvelle occasion pour ouvrir le livre saint et lire des extraits. Les déplacements d'Eléazar et sa famille ouvrent lieu aux placements textuels. La découverte aussi d'un nouveau monde, d'autres races humaines, le désert aride et le climat sec - qui contraste avec les pairies irlandaises vertes et humides -, d'autres cultures, d'autres mœurs... donnent naissance à des placements textuels, à des digressions.

Le déplacement est un terme qui, au sens propre, peut se définir comme: la mobilité ou le mouvement par lequel se réalise l'action de se déplacer, de changer de place, aller d'un lieu à un autre. Quant au placement c'est une entreprise qui est généralement lucrative, fructueuse, valorisante.

Pour ses déplacements imaginaires, les lectures effectuées par Michel Tournier ont eu une importance décisive. En effet, en passionné du voyage, il s'intéresse à tout : à l'Histoire, à la géographie (d'ailleurs à ce propos, Michel Tournier, lui- même se qualifie d' "écrivain géographe», mais pas seulement!), à la sociologie, aux mythes, aux légendes, aux cultures, à la peinture, à la photographie, bref à la vie.

Quant à la mise en scène textuelle, l'auteur essaie d'

explorer les possibilités de narration, à faire jouer les formes de représentation,

à saisir dans un même mouvement le lieu où l'on est et ses antipodes. $^{(1)}$

Le voyage peut prendre parfois une dimension pédagogique : essayer d'en savoir plus sur un pays, ses coutumes, ses traditions, la qualité de vie, comprendre le cycle de la vie chez un peuple, une ethnie...

Nous avons pu remarquer dans certains romans de Michel Tournier des déplacements géographiques en vue des voyages effectués par les héros, héros qui ne sont pas confinés dans des espaces statiques qui sont plutôt en déplacement permanent. Même Robinson dans Vendredi ou les limbes du Pacifique, qui, de prime abord, est sur une île plus de 28ans, ne cesse de se déplacer toujours à la découverte de choses nouvelles, exploitables, susceptibles de lui faciliter la vie sur l'île. Paul dans Les Météores, Idriss dans La Goutte d'or ainsi qu'Eléazar dans Eléazar ou la Source et le Buisson pour qui le déplacement est l'essence même du roman :

le voyage est l'occasion de déplacer de son milieu le personnage pour juger des effets de ce déplacement, à la fois en termes de connaissance du sujet (biographique) et des effets narratifs qu'il procure.$^{(2)}$ 
Se déplacer, c'est traverser des lieux, c'est aller dans un ailleurs chercher l'inconnu ou l'autre, voir ce que l'on ne peut trouver dans l'immobilité. Le déplacement au sens propre du terme présente un moment incomparable où l'on est poussé, par le désir ou par la contrainte, à s'exposer et à se frotter au dehors, à l'inconnu avec ses promesses et ses dangers. Se déplacer c'est d'abord suivre un chemin selon un procédé de mobilité, à la recherche de l'inconnu et parfois même du lointain.

Le terme d' " aventure » revient à chaque fois et avec insistance dès que l'on parle de déplacement, le mot est lourd de signification. L'aventure fascine l'homme parce qu'elle est chargée de mystère, de risques et de dangers, qui obligent l'être humain à se dépasser soi-même. Le déplacement est étroitement lié à l'aventure et à la découverte de la diversité étroite et sectaire, des peuples, des cultures, des communautés, et des conditions humaines.

Le déplacement peut aussi avoir des motivations psychologiques telles que le plaisir du dépaysement et le goût du changement afin d'échapper à un quotidien trop pesant. C'est le moyen assuré d'évasion. Il est souvent dépeint comme symbole d'ouverture sur l'autre et sur le monde, d'inclusion sociale, tandis que l'immobilité est associée à l'exclusion, la fixité, l'ancrage, l'immobilisme et l'enfermement.

Le déplacement dans ce cas n'est pas que physique, l'esprit aussi se déplace et suit le corps en mouvement. Le corps devient alors le moteur, l'instrument de déplacement, le véhicule du voyage.

Il y a une certaine perception de l'espace par le déplacement qui provoque l'imagination, le désir d'aventure et l'exploration de l'inconnu. Il en résulte aussi une exploration de soi et hors de soi. En psychanalyse, le déplacement est un mécanisme de défense qui déplace la valeur des choses et finalement le sens. Le déplacement alors, donne lieu aux placements textuels et discursifs, placement qui n'est pas seulement mise en place, mais aussi, au sens économique du terme, c'est-à-dire, action lucrative et valorisante. Le texte se retrouve donc enrichi par ces placements discursifs et le sens n'est plus qu'enrichi également.

Dans Vendredi ou les limbes du pacifique, le premier déplacement de Robinson c'est la traversée depuis l'Angleterre jusqu'à l'île, l'archipel da Juan Fernandez, Robinson va poursuivre son déplacement sur l'île elle-même, d'abord pour l'explorer, ensuite essayer d'en titrer profit pour sa survie : habits, abris, nourriture... ainsi que les possibilités de subsistance qui s'offrent à lui. Il était en constante mobilité au sein même de l'île, et à ce moment-là le déplacement devient aussi vital que le fait de survivre. Son seul moment de mobilité c'était quand il se ressourçait au fond de la grotte (l'Utérus d'Esperanza). Ses déplacements n'étaient pas régis par des règles mais il jouissait d'une liberté de mouvance. Parallèlement aux déplacements du 
personnage, l'auteur leur faisait correspondre des placements discursifs notamment les réflexions et remarques notées sur son Log-book :

Lorsqu'un peintre ou un graveur introduit des personnages dans un paysage ou à proximité d'un monument, ce n'est pas par goût de l'accessoire. Les personnages donnent l'échelle et, ce qui importe davantage encore, ils constituent des points de vue possibles qui ajoutent au point de vue réel de l'observateur d'indispensables virtualités. (p.53)

Pour Michel Tournier, c'est l'introduction de textes qui constitue des points de vue possibles ajoutés à son point de vue en tant qu'observateur.

Je pense que l'âme ne commence à avoir un contenu notable qu'au-delà du rideau de peau qui sépare l'intérieur de l'extérieur, et qu'elle s'enrichit indéfiniment à mesure qu'elle s'annexe des cercles plus vastes autour du point-moi. (p.70).

On ne sait plus s'il s'agit de l'âme de l'être humain ou celle du roman ?

Il y a ainsi deux problèmes de la connaissance, ou plutôt deux connaissances (...): la connaissance par autrui de la connaissance par moi-même. En mélangeant les deux sousprétextes qu'autrui est un autre moi, on n'aboutit à rien. (p.96).

Cette affirmation confirme, si besoin est, pourquoi l'auteur en fait étalage.

Exister qu'est-ce que ça veut dire ? Ça veut dire être dehors, sistere ex. Ce qui est à l'extérieur existe. Ce qui est à l'intérieur n'existe pas. Mes idées, mes images, me rêves n'existent pas. Si Speranza n'est qu'une sensation ou un faisceau de sensations, elle n'existe pas. Et moi-même je n'existe qu'en m'évadant de moi-même vers autrui. Ce qui complique tout, c'est que ce qui n'existe pas s'acharne à faire croire le contraire. Il y a une grande et commune aspiration de l'inexistant vers l'existence. C'est comme une force centrifuge qui pousserait vers le dehors tout ce qui remue en moi, images, rêveries, projets, fantasmes, désirs, obsessions. Ce qui n'exsiste pas in-siste. Tout ce petit monde se pousse à la porte du grand, du vrai monde. Et c'est autrui qui en tient la clef. (p.129)

C'est après que Robinson ait traversé une forêt et débouché sur un plateau de prairies que Tournier débat d'une question existentielle.

Le déplacement, la mouvance s'accompagne de placement textuel.

Dans Les Météores, les déplacements de Paul à travers le monde suscitent aussi des placements textuels à chaque lieu visité. Mais les déplacements de l'oncle de Paul, Alexandre, sont aussi accompagnés de placements textuels: un premier placement, une sorte d'ébauche de la psychosociologie du pauvre suite à son changement d'hôtel et du décor et qu'il 
va énumérer par article. Explication de ce qu'est un délinquant, explication donnée entre parenthèses. Une réflexion approfondie sur le Christ philosophiquement et théologiquement. L'endogamie et exogamie.

Réflexion sur la gémellité, l'éolien, le dialogue en marchant sur la plage. Sur les sans-pareil, le mot météore et sur Phileas Fogg du Tour du monde en quatre-vingts jours de Jules Verne, ainsi qu'un extrait du roman. La vision sur autrui.

Le premier déplacement de Paul étant Venise, Tournier ne pouvait pas outre passer un placement textuel sur les miroirs Vénitiens :

On enfile la calle Larga San Marco qui vient buter sur le rio di Palazzo qu'enjambe en aval le pont des soupirs. Le pont qui s'offre mène directement dans l'atelier du vieux Murano. C'est le royaume du verre. Au rez-de-chaussée devant des fours incandescents, les artisans verriers tournent au bout de leur longue canne la masse pâteuse, laiteuse, une énorme goutte irisée qui s'étire vers le sol dès que la rotation s'arrête. La canne est creuse. C'est une sorte de pipe, et l'ouvrier en soufflant dans cette pipe gonfle en ampoule, en bulle, en ballon. Ce spectacle déconcerte l'imagination, parce qu'il va à l'encontre de sa logique matérielle. (p.429)

Et, D'ailleurs, on assiste d'étape en étape à la naissance d'un flacon, d'une bouteille, d'une coupe, par des opérations aussi paradoxales que le refoulement du fond par un pontil, le modelage du goulot à la pince, le renforcement des bords par un bourrelet, l'adjonction d'un mince boudin qui devient entrelacs, torsade, tresse ou anse. (p.429)

La verrerie Murano et la fabrication de verre sont au cœur du récit. Il ne s'agit pas d'une simple observation, mais bien plus que cela, il est question de commentaires sur la fabrication, la matière et le produit finalisé :

Torturé et humilié au rez-de-chaussée par le feu, les cannes et les pinces, le verre ne retrouve son essence et sa souveraineté qu'au premier étage. Car le verre est froid, dur, cassant, brillant. Tels sont ses attributs fondamentaux. Pour le rendre souple, gras, et fumant, il faut le soumettre à d'épouvantables sévices. Dans ces salons d'exposition, il s'épanouit dans toute sa morgue glacée et maniérée. (p.429-430)

Il y a aussi l'histoire de Casanova : qui il était, son parcours, des évènements cocasses qui lui sont arrivés. La description de la station météorologique de Venise et l'essentiel de son travail. Les saisons et à quoi elles correspondent puis cela s'étend jusqu'à l'œuvre Les Saisons de Vivaldi.

Le voyage, déplacement dans l'espace, est une affaire de temps en allant en Islande. La gémellité dépariée et son ubiquité qu'est le voyage.

Le japon sans les jardins japonais : 
le jardin, la maison et l'homme sont un organisme vivant qu'il ne faut pas démembrer(...) le jardin et la maison doivent se mêler intimement l'un à l'autre. Les jardins occidentaux ignorent cette loi(...) la maison du sage invertit le jardin par une suite de bâtiments légers montés sur des piliers de bois eux-mêmes posés sur des pierres plate. Des panneaux coulissants, tantôt opaques, tantôt translucides, y dessinent un espace mouvant qui rend inutile les portes et les fenêtres. Le jardin et la maison se baignent dans la même lumière. Dans la maison japonaise traditionnelle, il ne saurait y avoir des courants d'air, il n'y a que du vent. La maison par un réseau de passerelles et de galeries paraît se diluer dans le jardin. En vérité on ne sait lequel des deux envahit et absorbe l'autre, c'est plus qu'un mariage heureux. C'est le même être.

Les pierres ne doivent jamais être simplement posées sur le sol. Il faut qu'elles soient toujours quelque peu enfouies. Car la pierre possède une tête, une queue, un dos, et son ventre à besoin de la chaude obscurité de la terre. (p.517-518).

Et s'en suit tout un savoir sur les pierres, les animaux et encore les jardins mais, il s'agit cette fois-ci, d'un genre particulier, le jardin Zen! L'auteur explique ce qu'est un jardin Zen, son agencement, son architecture, sa disposition...

Nous retrouvons également une digression sur Le Pacte de Varsovie du 12 aout 1961, sur la langue islandaise et sur la cryptophasie dépariée. Arrivé à Vancouver, description détaillée de cette ville: la faune, la flore, les constructions, les gens, la vie. La description s'étend jusqu'au train du Canadian Pacific Railway.

Il y a aussi une digression sur l'Histoire : l'Allemagne de l'Est et l'Allemagne de l'Ouest, le mur de Berlin, le discours du conseil des ministres de la R.D.A. qui est transcrit sur plusieurs pages. Toutes ces références, ces précisions donnent à lire un nouveau texte : un document de l'Histoire.

Dans La Goutte d'or, les déplacements géographiques sont aussi accompagnés de placements discursifs : le premier déplacement/placement se produit lors de la fête de mariage à laquelle assiste Idriss, Zett Zobeida qui commence aussi à bouger au rythme de la danse et une litanie de vers se glisse au texte et se répètera tout au long du roman, et à chaque fois qu'Idriss sera en mouvement : le lendemain du mariage en partant à la recherche de sa photo, sur le car-ferry en partance pour Marseille, le train depuis Marseille jusqu'à Paris.

Aussi, l'histoire de Barberousse ou Le portrait du roi est racontée quand Idriss va à un mariage et ce après la danse de Zett Zobeida. La fabrication des mannequins en allant à Pantin à l'usine de Glyptoplastique, la mort du général Laperrine quand il va dans un restaurant typiquement maghrébin, la vie de la 
chanteuse Oum Keltoum lorsqu'il est au foyer de la rue Myra. L'art de la calligraphie dans l'atelier du maître Abd Al Ghafari et enfin le conte de La Reine blonde chez le maître calligraphe.

Dans Eléazar ou la Source et le Buisson, le placement textuel débute quand Eléazar entre au séminaire gallican en Ulster avec l'histoire de saint Patrick, l'évangélisateur de l'Irlande, dont a été rapportée l'histoire par Jacques de Voragine dans sa Légende dorée. Juste après, c'est un extrait de ce mot de Jésus à la Samaritaine sur la margelle du puits de Jacob, tiré de saint Jean, IV, 14.

Ensuite c'est l'allusion de l'Evangile de Matthieu, XVIII, 10.

A chaque pas et à chaque fois qu'il doit prendre une décision, Eléazar sort sa Bible et lit un passage et fait son choix. Le livre saint devient sa référence, sa carte géographique ; c'est le guide suprême et spirituel. Tout est écrit et assuré par la parole divine, juste et sage. Le déplacement de Eléazar donne lieu à des placements textuels, bibliques ou autre car parfois Eléazar se rappelle des textes lus lors de sa formation de pasteur sur Moïse ou le peuple hébreux qui confirme son idée initiale (son destin et celui de Moïse sont très liés). Depuis son départ de son Irlande natale, plus précisément le port de Cork jusqu'au Nouveau Monde. A Portsmouth en Virginie, il lit des passages de la Bible pour choisir sa destination, ensuite de l'Ohio en passant par Cincinnati, Saint Louis, le Colorado, le Nevada et enfin la Californie.

Dans le désert américain, l'apprentissage de la vie et de la vérité sur l'histoire de Moïse et le peuple hébreux sous l'emprise du joug égyptien devient plus qu'une évidence. Moïse affrontant le Pharaon, les quarante jours de la traversée de Moïse et de son peuple et les six semaines de la traversée d'Eléazar et sa famille jusqu'en Amérique, le Nouveau Monde. Tout est écrit : plus Eléazar se projette dans le passé et la vie de Moïse, plus les ressemblances sont frappantes.

Donc, ces déplacements des héros donnent lieu à des «placements » textuels et discursifs. Plus exactement, cela donne lieu à des digressions. C'est un voyage de l'écriture grâce à l'imaginaire. Un imaginaire qui encode la langue d'écriture au profit d'une nouvelle forme, un nouveau style d'écriture. En ce sens, la digression peut être en écriture comme procédé au service de l'intrigue et passer de la transgression à l'intégration en y laissant des réminiscences intertextuelles. Ce procédé fait appel à une nouvelle prose qui sera le lieu de rencontre de plusieurs types et genres de discours en un seul. Non pas que le roman est devenu un fourre-tout discursif mais l'intégration de tous ces types de discours se fait à l'aide d'outils et de procédés qui réfléchissent (réfléchissent dans les deux sens) un genre autre. Le texte soumis au lecteur se donne à lire comme un tout complexe et créatif.

\section{Bibliographie :}


- BARTHES Roland, Théorie $d u$ texte, Article in Encyclopédie universelle, 1973

- BARTHES Roland, Introduction à l'analyse structurale des récits, poétique du récit, Paris, le Seuil, 1981.

- BARTHES Roland, Le degré zéro de l'écriture suivi de Nouveaux essais critiques, Paris, Seuil, 1972.

- BENACHOUR-TEBBOUCHE Nedjma, Constantine et ses écrivainsvoyageurs, Constantine, ed Chihab, 2015.

- BENACHOUR-TEBBOUCHE Nedjma, Constantine et ses romanciers, Constantine, ed Média-Plus, 2008 . p.231.

- BOURDIEU Pierre, Langage et pouvoir symbolique, Paris, Fayard, 2001.

- BOURDIEU Pierre, Les règles de l'art, Genèse et structure du champ littéraire, Paris, Seuil, 1992.

- CHAUlET-ACHOUR Christiane, BEKKAT Amina, Clefs pour la lecture des récits : Convergences critiques II, Ed.Tell, 2002.

- DALLENBACH Lucien, Le Récit spéculaire, Essai sur la mise en abyme, Paris, Seuil, 1977.

- DERUELLE Aude, Balzac et la digression. Une nouvelle prose romanesque. Saint-Cyr sur Loire, Christian Pirot Editeur, 2004.

- ECO Umberto, Lector in fabula, le rôle du lecteur ou la coopération interprétative dans les textes narratifs, Paris, Grasset et Fasquelle, 1985.

- ESCARPIT Robert, Le littéraire et le social, Eléments pour une sociologie de la littérature, Paris, Flammarion, 1970.

- GENETTE Gérard, Figure III, Paris, Seuil, 1972.

- GENETTE Gérard, Introduction à l'architexte, Paris, Seuil, 1979.

- GENETTE Gérard, Nouveau discours du récit, Paris, Seuil, 1983.

- GENETTE Gérard, Palimpsestes, Paris, Le Seuil, 1982.

- GOLDENSTEIN Jean-Pierre, Pour lire le roman, Paris, Duculot, 1985.

- KERBRAT-ORECCHIONI Catherine, L'implicite, Paris, Armand Colin, 1985.

- $\quad$ LUCKAS Georges, La théorie du roman, Paris, Denoël, 1968.

- MACHEREY Pierre, Pour une théorie de la production littéraire, Paris, Maspero, 1966. ?

- SABRY Randa, Stratégies discursives : Digression, transition, suspens, Paris, Ed. Ecoles des Hautes Etudes en Sciences Sociales, 1992. 
- TOURNIER Michel, Vendredi ou les Limbes du Pacifique, Paris, Gallimard, 1967.

- TOURNIER Michel, Les Météores, Gallimard, 1975

- TOURNIER Michel, La Goutte d'Or, Paris, Gallimard, 1985.

- TOURNIER Michel, Eléazar ou la Source et le Buisson, Paris, Gallimard, 1996.

- VIVIES Jean, Le récit de voyage en Angleterre au XVIIe siècle, De l'inventaire à l'invention, Presses universitaires du Mirail, 1999.

Note :

${ }^{1}$ Jean Roudaut in Encyclopédie Universalis, SA, 1995. In, BENACHOURTEBBOUCHE Nedjma, Constantine et ses écrivains-voyageurs, Constantine, ed Chihab, 2015 . p. 3.

${ }^{2}$ Viviès Jean, Le récit de voyage en Angleterre au XVIIe siècle, De l'inventaire à l'invention, Presses universitaires du Mirail, 1999.p.72 